\title{
Lernen und Identität
}

\author{
Nils Bernhardsson-Laros
}

Online publiziert: 11. März 2015

(c) Die Autor(en) 2015. Dieser Artikel ist auf Springerlink.com mit Open Access verfügbar.

\section{Rezension zu:}

Knud Illeris (2014). Transformative Learning and Identity. Routledge, Abingdon und New York, 177 Seiten, 50,27 €, ISBN 978-0-415-83890-0

Im Zentrum des Buches steht Transformatives Lernen (TL) nach Jack Mezirow. Die Theorie zielt darauf ab, die Spezifika des Lernens von Erwachsenen zu beschreiben. Dabei richtet sie den Blick nicht in erster Linie auf das Erlernen von Wissen, sondern auf die Bemühungen der Menschen um die Weiterentwicklung ihres Denkens, Fühlens und Handelns, womit sie in eine ähnliche Richtung weist wie die Bildungstheorie.

Illeris versteht sein Buch als einen Beitrag, der sich vor allem um die wissenschaftliche Anschlussfähigkeit des TL bemüht (S. xi ff.). Der Autor macht deutlich, dass die vermeintliche Stärke der Theorie, ihre Offenheit, zugleich auch zur Gefahr für die Theorie werden kann. Illeris unterscheidet zwischen der Theorie des TL und einer sozialen Bewegung (,movement"), welche sich um das TL herum gebildet hat (S. 14 f.). Er diagnostiziert, dass die soziale Bewegung die unterschiedlichsten Akteure zusammenführt, welche oftmals eine Überinterpretation der theoretischen Grundlagen des TL vornehmen und jegliche Veränderungen und neue Erfahrungen als TL interpretieren. Die Unklarheiten in der theoretischen Anlage des TL führt Illeris auf den für die Theorie grundlegenden Begriff des Selbst (,the learner's self“) zurück und schlägt vor, diesen durch den Begriff der Identität (,,identity“) zu ersetzen, um zu einem eindeutigeren Verständnis der Theorie zu gelangen (S. xiii).

Illeris gliedert sein Buch in drei Teile. Im ersten Teil begründet er in einem historischen Zugriff, warum es aus seiner Sicht notwendig ist, eine „Redefinition of

\footnotetext{
N. Bernhardsson-Laros $(\varangle)$

Fachhochschule Nordwestschweiz (FHNW),

Windisch, Schweiz

E-Mail: nils.berhardssonlaros@fhnw.ch
} 
transformative learning“" (S. 38) vorzunehmen und führt seine eigene Definition zum TL ein, in deren Zentrum nicht die Transformation des „learner's self“ (S. 40) steht, sondern die der Identität der Lernenden.

In Teil zwei arbeitet Illeris mit Bezug auf Erikson sowie auf soziologische Fundierungen des Identitätsbegriffes heraus, dass er Identität als psycho-soziales Konzept versteht. Er geht davon aus, dass Menschen im Laufe ihrer Kindheit und Jugend eine „core-identity“ (S. 70) ausbilden, die relativ stabil ist und ihnen dabei hilft, ihre Interaktionen mit ihrer Umwelt zu koordinieren. Die Kernidentität ist von einem ,layer of personality“ (S. 72) umgeben. Hier verortet Illeris transformative Erfahrungen der kognitiven Art, wie sie Mezirow beschreibt. Kernidentität und Persönlichkeit werden wiederum von einem ,layer of preferences“ (S. 73) gerahmt. Auf dieser Ebene entscheidet das Individuum situativ darüber, ob die Dinge, die im alltäglichen Leben seine Aufmerksamkeit beanspruchen, Anlass geben für eine Auseinandersetzung mit der eigenen Identität.

Im dritten Teil beleuchtet Illeris seine Verbindung von TL und Identität, indem er auf Beispiele aus verschiedenen schulischen und außerschulischen Erziehungsund Bildungskontexten sowie dem Arbeitsleben Bezug nimmt. Interessant ist dabei vor allem seine Auseinandersetzung mit der Kompetenzdebatte. Er entwickelt das Argument, dass das Kompetenzkonzept in der dominierenden Debatte um Kompetenzmessung auf ein Verständnis einer administrativen und nahezu vollständig formalisierten Kategorie enggeführt wird, weshalb es eine mehr als zweifelhafte Basis für die Einstufung und Auswahl von Arbeitskräften darstelle. Illeris betont, dass die volitionalen Voraussetzungen zur Entwicklung von Kompetenzen im Rahmen transformativer Lernprozesse, die in der Identität gründen und daher nicht messbar sind, in der Kompetenzdebatte bisher weitgehend unberücksichtigt bleiben.

In kritischer Hinsicht fällt auf, dass es Illeris nicht vollständig gelingt, den Begriff des Selbst durch den Begriff der Identität zu ersetzen. Dies wird besonders im letzten Kapitel deutlich, in dem er zusammenfassend auf das Thema ,identity and identity development“" (S. 145) eingeht: ,(I)individualization implies that the individual identity - understood as the experience of the self, and externally in relation to others - gradually has become extremely important" (ebd.). Demnach benötigt die Identität immer noch einen Gegenbegriff, mit dem die Identitätsarbeit, die das Individuum vollzieht, erklärt werden kann. Dieser scheint nach wie vor das Selbst zu sein, welches in unterschiedlichen Lebenssituationen in Erfahrung gebracht wird. Dies legt nahe zu fragen, ob es nicht etwas voreilig war, den einen Term vollständig durch den anderen ersetzen zu wollen. Abgesehen von dieser Unschärfe handelt es sich um einen gelungenen Vorschlag einer exakteren Fassung der Theorie des TL. Illeris eröffnet damit die Debatte um eine umfassende Neuinterpretation des TL und es bleibt abzuwarten, ob neben Kritik und möglichen Ergänzungen auch Alternativvorschläge unterbreitet werden. Insgesamt ist das Buch aufgrund der vielen Praxisbeispiele sehr anschaulich gestaltet, weshalb es auch für eine praxisorientierte Leserschaft, die sich mit dem TL vertraut machen will und sich davon Anregungen für ihr alltägliches professionelles Handeln erwartet, eine spannende Lektüre darstellt. 
Open Access Dieser Artikel unterliegt den Bedingungen der Creative Commons Attribution License. Dadurch sind die Nutzung, Verteilung, und Reproduktion erlaubt, sofern der/die Originalautor/en und die Quelle angegeben sind. 